\title{
Investigation on the effect of beating on the physical and mechanical properties of untreated kenaf based insulation paper
}

\begin{abstract}
This paper presents a study on the effect of beating on the physical and mechanical properties of untreated kenaf based insulation paper for application in transformers. The paper was developed based on kenaf bast fibers through soda pulping process. The pulps were subjected to beating process up to 9000 revolutions where the thickness, density and tensile index of paper were measured. It is found that the tensile index and the density of the paper increases while the thickness of the paper decreases with beating revolutions.
\end{abstract}

Keyword: Kenaf based insulation paper; Tensile index; Transformers; Beating 